\title{
Single Swarm and Simple Multi-Swarm PSO Comparison
}

\author{
Michal Pluhacek ${ }^{1}$, Roman Senkerik ${ }^{1}$, Adam Viktorin ${ }^{1}$ and Ivan Zelinka ${ }^{2}$ \\ ${ }^{1}$ Faculty of Applied informatics \\ Tomas Bata University in Zlin, Nam T.G. Masaryka 5555, 760 01Zlin, Czech Republic \\ \{pluhacek, senkerik, aviktorin\}@fai.utb.cz \\ ${ }^{2}$ Department of Computer Science, Faculty of Electrical Engineering and Computer Science \\ VSB-TUO, 17.listopadu 15, 70833 Ostrava-Poruba, Czech Republic \\ ivan.zelinka@vsb.cz
}

\begin{abstract}
This paper presents a comparative study of the original single swarm PSO with linear decreasing inertia weight and basic multi-swarm variant. The alternative population topology (multi-swarms) is a topic that is getting increased attention from the research community in recent years. We present evidence that it might be very beneficial to divide the population into two subswarms with partially restricted communication. The size of the sub-swarms is chosen with respect to a previously published study on this topic. The scaling of the methods is compared in three different dimensional settings. The results are statistically evaluated and discussed. The paper concludes with proposals for future research.
\end{abstract}

Keywords: particle swarm optimization, swarm intelligence, multi-swarm, topology

\section{Introduction}

In the past decades, the Particle swarm optimization (PSO) algorithm has established itself as a very efficient global optimizer. The original design (Kennedy and Eberhart, 1995; Kennedy, 1997) has been regularly modified (Shi and Eberhart, 1998; Engelbrecht 2010) and studied in great detail (van den Bergh and Engelbrecht 2006).

One of the most popular approaches for PSO modification is the multi-swarm (Liang and Suganthan, 2005; Ostadrahimi et al. 2012; Solomon et al., 2011; Dor, 2012; Liu et al., 2011) approach. In the multiswarm approaches, the population is divided into multiple sub-populations (sub-swarms) with different levels of communication. The benefit of such approach is in that the population can maintain divergence, search multiple promising regions and partially converge into multiple optima.

In (García-Nieto and Alba, 2012) the optimal swarm (sub-swarm) size is discussed in great detail. It is proposed that six particles per swarm might be the optimal number for PSO based algorithms.
In this study, we build on those findings and compare the performance of single swarm PSO with a PSO with two sub-swarms that consist of six particles each.

The rest of the paper is structured as follows: In the following section, the PSO algorithm is described. In the next section, the proposed simple multi-swarm algorithm is introduced. Used test functions in this work are presented in the following section. The experiment is set up and results presented in the following sections. The paper concludes with the discussion of the results.

\section{Particle Swarm Optimization (PSO)}

The PSO algorithm is inspired by the natural swarm behavior of animals (such as birds and fish). It was firstly introduced by Eberhart and Kennedy (1995). Soon the PSO became a popular method for global optimization. Each particle in the population represents a possible solution of the optimization problem which is defined by the cost function (CF). In each iteration of the algorithm, a new location (combination of $\mathrm{CF}$ parameters) of the particle is calculated based on its previous location and velocity vector (velocity vector contains particle velocity for each dimension).

According to the method of selection of the swarm or subswarm for best solution information spreading, the PSO algorithms are noted as global PSO (GPSO) or local PSO (LPSO). Within this research, the PSO algorithm with global topology was utilized. The velocity calculation formula is given by

$$
\begin{aligned}
& v_{i j}^{t+1}=w \cdot v_{i j}^{t}+c_{1} \cdot \text { Rand } \cdot\left(\text { pBest }_{i j}-x_{i j}^{t}\right) \\
& +c_{2} \cdot \text { Rand } \cdot\left(\text { gBest }_{j}-x_{i j}^{t}\right)
\end{aligned}
$$

where

$v_{i j}^{t+1}$ - New velocity of the ith particle in iteration $t+1$. (component $j$ of the dimension $D$ ).

$w$ - Inertia weight value.

$v_{i j}{ }^{t}$ - Current velocity of the ith particle in iteration $t$. (component $j$ of the dimension $D$ ).

$c_{1}, c_{2}$ - Acceleration constants.

pBest $t_{i j}$ - Local (personal) best solution found by the ith particle. (component $j$ of the dimension $D$ ). 
$g B e s t_{j}$ - Best solution found in a population. (component $j$ of the dimension $D$ ).

$x_{i j}{ }^{t}$ - Current position of the ith particle (component $j$ of the dimension $D$ ) in iteration $t$.

Rand - Pseudo random number, interval $(0,1)$.

The maximum velocity was limited to $20 \%$ of the dimension range as it is usual. The new position of each particle is then given by:

$$
x_{i}^{t+1}=x_{i}^{t}+v_{i}^{t+1}
$$

where $x_{i}^{t+1}$ is the new particle position

Finally, the linear decreasing inertia weight ((Shi and Eberhart, 1998) is used in the typically referred PSO design that was used in this study. The dynamic inertia weight is meant to slow the particles over time thus to improve the local search capability in the later phase of the optimization. The inertia weight has two control parameters $w_{\text {start }}$ and $w_{\text {end }}$. A new $w$ for each iteration is given by (3), where $t$ stands for current iteration number and $n$ stands for the total number of iterations. The values used in this study were $w_{\text {start }}=0.9$ and $w_{\text {end }}=0.4$.

$$
w=w_{\text {start }}-\frac{\left(\left(w_{\text {start }}-w_{\text {end }}\right) \cdot t\right)}{n}
$$

\section{Proposed multi-swarm model}

In this initial study, a simple multi-swarm PSO was utilized. In the proposed approach the population is divided into two subswarms. Each subswarm utilizes different $\mathrm{gBest}$ for particle movement. After every 100 iterations, the values of $g B e s t$ of swarm 1 and gBest of swarm 2 are compared, and the gBest with better value is copied as a gBest for both swarms.

Using this simple method, the population-wide communication is restricted to periodical timewindows.

\section{Test Functions}

Within this initial research, four well-known and frequently used benchmark functions were utilized. The optimum value is 0 for all functions. Four benchmark functions are used in the comparisons.

\section{Sphere function}

$$
f_{1}(x)=\sum_{i=1}^{D} x_{i}^{2}
$$

where D - dimension

Search Range: $[-100,100]^{\mathrm{D}}$

Glob. Opt. Pos.: [0]

\section{Schwefel's function}

$$
f_{5}(x)=418.9829 \cdot \mathrm{D} \sum_{i=1}^{D}-x_{i} \sin (\sqrt{|x|})
$$

Search Range: $[-500,500]^{\mathrm{D}}$

Glob. Opt. Pos.: [420.96]

\section{Rastrigin's function}

$$
f_{6}(x)=\sum_{i=1}^{D}\left[x_{i}^{2}-10 \cos \left(2 \pi x_{i}\right)+10\right]
$$

Search Range: $[-5.12,5.12]^{\mathrm{D}}$

Glob. Opt. Pos.: [0]

\section{Rosenbrock's function}

$$
f_{3}(x)=\sum_{i=1}^{D-1}\left[100\left(x_{i}^{2}-x_{i+1}\right)^{2}+\left(1-x_{i}\right)^{2}\right]
$$

Search Range: $[-10,10]^{\mathrm{D}}$

Glob. Opt. Pos.: $[0]^{\mathrm{D}}$

\section{Experiment setup}

Two variants of the PSO algorithm were compared in this study with 3 different dimension settings. The single-swarm PSO with linear decreasing inertia weight (as described in section 2) noted further PSO. The multiswarm variant proposed in section 3 is noted further PSO Multi.

The control parameters were set as follows:

Population size: 12 (PSO) 6+6 (PSO Multi)

Iterations: 2500

$v_{\max }: 0.2$

$\mathrm{W}_{\text {start: }} 0.9$

$\mathrm{W}_{\text {end }}: 0.4$

$\mathrm{c}_{1}, \mathrm{c}_{2}=2$

$\operatorname{Dim}=5,10,20$

\section{Results}

In this section results obtained in the experiments with both previously described PSO variants are presented and compared. In table $1-12$ the statistical overview of results from 30 repeated runs of each algorithm are given alongside with the p-value of Wilcoxon signedrank test with significance level 0.05 . The best mean results are highlighted by bold numbers. 
Table 1. Results - Sphere function: dim: 5 .

\begin{tabular}{|l|r|r|r|}
\hline $\begin{array}{l}\text { Sphere } \\
\text { dim: 5 }\end{array}$ & PSO & PSO Multi & p-value \\
\hline $\begin{array}{l}\text { Mean CF } \\
\text { Value: }\end{array}$ & $2.051 \mathrm{E}-14$ & $\mathbf{1 . 1 3 6 E - 8 8}$ & \multirow{2}{*}{$1.825 \mathrm{E}-06$} \\
\hline Std. Dev.: & $1.123 \mathrm{E}-13$ & $5.367 \mathrm{E}-88$ & \\
\hline $\begin{array}{l}\text { CF Value } \\
\text { Median: }\end{array}$ & $6.307 \mathrm{E}-31$ & $8.659 \mathrm{E}-93$ & \\
\cline { 1 - 2 } $\begin{array}{l}\text { Max, CF } \\
\text { Value: }\end{array}$ & $6.152 \mathrm{E}-13$ & $2.928 \mathrm{E}-87$ & \\
\cline { 1 - 2 } $\begin{array}{l}\text { Min, CF } \\
\text { Value: }\end{array}$ & $1.914 \mathrm{E}-45$ & $4.884 \mathrm{E}-98$ \\
\cline { 1 - 2 }
\end{tabular}

Table 2. Results - Schwefel function: dim: 5.

\begin{tabular}{|c|c|c|c|}
\hline $\begin{array}{l}\text { Schwefel } \\
\text { dim: } 5\end{array}$ & PSO & PSO Multi & p-value \\
\hline $\begin{array}{l}\text { Mean CF } \\
\text { Value: }\end{array}$ & $4.106 \mathrm{E}+02$ & $2.645 \mathrm{E}+02$ & $9.280 \mathrm{E}-04$ \\
\hline Std. Dev.: & $1.697 \mathrm{E}+02$ & $1.344 \mathrm{E}+02$ & \\
\hline $\begin{array}{l}\text { CF Value } \\
\text { Median: }\end{array}$ & $4.540 \mathrm{E}+02$ & $2.369 \mathrm{E}+02$ & \\
\hline $\begin{array}{l}\text { Max, CF } \\
\text { Value: }\end{array}$ & $7.896 \mathrm{E}+02$ & $5.922 \mathrm{E}+02$ & \\
\hline $\begin{array}{l}\text { Min, CF } \\
\text { Value: }\end{array}$ & $1.184 \mathrm{E}+02$ & $6.364 \mathrm{E}-05$ & \\
\hline
\end{tabular}

Table 3. Results - Rastrigin function: dim: 5.

\begin{tabular}{|l|r|r|r|}
\hline $\begin{array}{l}\text { Rastrigin } \\
\text { dim: 5 }\end{array}$ & PSO & PSO Multi & p-value \\
\hline $\begin{array}{l}\text { Mean CF } \\
\text { Value: }\end{array}$ & $2.056 \mathrm{E}+00$ & $\mathbf{3 . 9 8 0 E - 0 1}$ & \multirow{2}{*}{$4.072 \mathrm{E}-06$} \\
\hline Std. Dev.: & $1.522 \mathrm{E}+00$ & $5.604 \mathrm{E}-01$ & \\
\hline $\begin{array}{l}\text { CF Value } \\
\text { Median: }\end{array}$ & $1.990 \mathrm{E}+00$ & $0.000 \mathrm{E}+00$ & \\
\cline { 1 - 2 } $\begin{array}{l}\text { Max, CF } \\
\text { Value: }\end{array}$ & $5.970 \mathrm{E}+00$ & $1.990 \mathrm{E}+00$ & \multirow{2}{|}{} \\
\cline { 1 - 2 } $\begin{array}{l}\text { Min, CF } \\
\text { Value: }\end{array}$ & $2.842 \mathrm{E}-14$ & $0.000 \mathrm{E}+00$ \\
\cline { 1 - 2 }
\end{tabular}

Table 4. Results - Rosenbrock function: dim: 5 .

\begin{tabular}{|c|c|c|c|}
\hline $\begin{array}{l}\text { Rosenbrock } \\
\text { dim: } 5\end{array}$ & PSO & PSO Multi & p-value \\
\hline $\begin{array}{l}\text { Mean CF } \\
\text { Value: }\end{array}$ & $2.621 \mathrm{E}-01$ & $1.389 \mathrm{E}-01$ & $3.732 \mathrm{E}-04$ \\
\hline Std. Dev.: & $9.973 \mathrm{E}-01$ & 4.911E-02 & \\
\hline $\begin{array}{l}\text { CF Value } \\
\text { Median: }\end{array}$ & $1.344 \mathrm{E}-19$ & $1.273 \mathrm{E}-01$ & \\
\hline $\begin{array}{l}\text { Max, CF } \\
\text { Value: }\end{array}$ & $3.931 \mathrm{E}+00$ & $2.496 \mathrm{E}-01$ & \\
\hline $\begin{array}{l}\text { Min, CF } \\
\text { Value: }\end{array}$ & $0.000 \mathrm{E}+00$ & $6.849 \mathrm{E}-02$ & \\
\hline
\end{tabular}

Table 5. Results - Sphere function: dim: 10 .

\begin{tabular}{|l|r|r|r|}
\hline $\begin{array}{l}\text { Sphere } \\
\text { dim: 10 }\end{array}$ & PSO & PSO Multi & p-value \\
\cline { 1 - 2 } $\begin{array}{l}\text { Mean CF } \\
\text { Value: }\end{array}$ & $4.586 \mathrm{E}-04$ & $\mathbf{2 . 6 3 5 E - 4 1}$ & \multirow{2}{*}{$1.825 \mathrm{E}-06$} \\
\hline Std. Dev.: & $4.364 \mathrm{E}-04$ & $6.049 \mathrm{E}-41$ & \multirow{2}{|}{} \\
\hline $\begin{array}{l}\text { CF Value } \\
\text { Median: }\end{array}$ & $2.739 \mathrm{E}-04$ & $1.185 \mathrm{E}-42$ & \\
\cline { 1 - 2 } $\begin{array}{l}\text { Max, CF } \\
\text { Value: }\end{array}$ & $1.737 \mathrm{E}-03$ & $2.155 \mathrm{E}-40$ & \\
\cline { 1 - 2 } $\begin{array}{l}\text { Min, CF } \\
\text { Value: }\end{array}$ & $3.916 \mathrm{E}-06$ & $1.328 \mathrm{E}-45$ & \\
\cline { 1 - 2 } & &
\end{tabular}

Table 6. Results - Schwefel function: dim: 10 .

\begin{tabular}{|c|c|c|c|}
\hline $\begin{array}{l}\text { Schwefel } \\
\text { dim: } 10\end{array}$ & PSO & PSO Multi & p-value \\
\hline $\begin{array}{l}\text { Mean CF } \\
\text { Value: }\end{array}$ & $1.443 \mathrm{E}+03$ & $8.751 \mathrm{E}+02$ & $2.237 \mathrm{E}-06$ \\
\hline Std. Dev.: & $2.209 \mathrm{E}+02$ & $2.307 \mathrm{E}+02$ & \\
\hline $\begin{array}{l}\text { CF Value } \\
\text { Median: }\end{array}$ & $1.423 \mathrm{E}+03$ & $8.982 \mathrm{E}+02$ & \\
\hline $\begin{array}{l}\text { Max, CF } \\
\text { Value: }\end{array}$ & $2.057 \mathrm{E}+03$ & $1.323 \mathrm{E}+03$ & \\
\hline $\begin{array}{l}\text { Min, } \mathrm{CF} \\
\text { Value: }\end{array}$ & $1.052 \mathrm{E}+03$ & $3.356 \mathrm{E}+02$ & \\
\hline
\end{tabular}

Table 7. Results - Rastrigin function: dim: 10.

\begin{tabular}{|c|c|c|c|}
\hline $\begin{array}{l}\text { Rastrigin } \\
\text { dim: } 10\end{array}$ & PSO & PSO Multi & p-value \\
\hline $\begin{array}{l}\text { Mean CF } \\
\text { Value: }\end{array}$ & $9.356 \mathrm{E}+00$ & $4.676 \mathrm{E}+00$ & $3.561 \mathrm{E}-05$ \\
\hline Std. Dev.: & $2.472 \mathrm{E}+06$ & $2.864 \mathrm{E}+00$ & \\
\hline $\begin{array}{l}\text { CF Value } \\
\text { Median: }\end{array}$ & $9.268 \mathrm{E}+00$ & $3.980 \mathrm{E}+00$ & \\
\hline $\begin{array}{l}\text { Max, CF } \\
\text { Value: }\end{array}$ & $1.413 \mathrm{E}+01$ & $1.492 \mathrm{E}+01$ & \\
\hline $\begin{array}{l}\text { Min, CF } \\
\text { Value: }\end{array}$ & $2.351 \mathrm{E}+00$ & $9.950 \mathrm{E}-01$ & \\
\hline
\end{tabular}

Table 8. Results - Rosenbrock function: dim: 10 .

\begin{tabular}{|c|c|c|c|}
\hline $\begin{array}{l}\text { Rosenbrock } \\
\text { dim: } 10\end{array}$ & PSO & PSO Multi & p-value \\
\hline $\begin{array}{l}\text { Mean CF } \\
\text { Value: }\end{array}$ & $7.560 \mathrm{E}+00$ & $3.743 E+00$ & 4.502E-06 \\
\hline Std. Dev.: & $2.194 \mathrm{E}+06$ & $9.581 \mathrm{E}-01$ & \\
\hline $\begin{array}{l}\text { CF Value } \\
\text { Median: }\end{array}$ & $7.805 \mathrm{E}+00$ & $3.683 \mathrm{E}+00$ & \\
\hline $\begin{array}{l}\text { Max, CF } \\
\text { Value: }\end{array}$ & $1.519 \mathrm{E}+06$ & $8.144 \mathrm{E}+00$ & \\
\hline $\begin{array}{l}\text { Min, CF } \\
\text { Value: }\end{array}$ & $2.619 \mathrm{E}+00$ & $1.184 \mathrm{E}+06$ & \\
\hline
\end{tabular}

Table 9. Results - Sphere function: dim: 20 .

\begin{tabular}{|c|c|c|c|}
\hline $\begin{array}{l}\text { Sphere } \\
\text { dim: } 20\end{array}$ & PSO & PSO Multi & p-value \\
\hline $\begin{array}{l}\text { Mean CF } \\
\text { Value: }\end{array}$ & $6.930 \mathrm{E}-02$ & 8.433E-17 & $1.825 \mathrm{E}-06$ \\
\hline Std. Dev.: & 4.192E-02 & $1.890 \mathrm{E}-16$ & \\
\hline $\begin{array}{l}\text { CF Value } \\
\text { Median: }\end{array}$ & $5.756 \mathrm{E}-02$ & $1.003 \mathrm{E}-17$ & \\
\hline $\begin{array}{l}\text { Max, CF } \\
\text { Value: }\end{array}$ & $1.705 \mathrm{E}-01$ & $8.765 \mathrm{E}-16$ & \\
\hline $\begin{array}{l}\text { Min, CF } \\
\text { Value: }\end{array}$ & $2.370 \mathrm{E}-02$ & $8.788 \mathrm{E}-20$ & \\
\hline
\end{tabular}

Table 10. Results - Schwefel function: dim: 20 .

\begin{tabular}{|l|r|r|r|}
\hline $\begin{array}{l}\text { Schwefel } \\
\text { dim: 20 }\end{array}$ & PSO & PSO Multi & p-value \\
\cline { 1 - 2 } $\begin{array}{l}\text { Mean CF } \\
\text { Value: }\end{array}$ & $4.035 \mathrm{E}+03$ & $\mathbf{2 . 4 5 2 E + 0 3}$ & \multirow{2}{*}{$1.825 \mathrm{E}-06$} \\
\hline Std. Dev.: & $3.237 \mathrm{E}+02$ & $3.954 \mathrm{E}+02$ & \\
\hline $\begin{array}{l}\text { CF Value } \\
\text { Median: }\end{array}$ & $3.996 \mathrm{E}+03$ & $2.497 \mathrm{E}+03$ & \\
\cline { 1 - 2 } $\begin{array}{l}\text { Max, CF } \\
\text { Value: }\end{array}$ & $4.566 \mathrm{E}+03$ & $3.079 \mathrm{E}+03$ & \\
\cline { 1 - 2 } $\begin{array}{l}\text { Min, CF } \\
\text { Value: }\end{array}$ & $3.335 \mathrm{E}+03$ & $1.796 \mathrm{E}+03$ & \\
\cline { 2 - 3 } & \multicolumn{2}{|l}{} \\
\end{tabular}


Table 11. Results - Rastrigin function: dim: 20.

\begin{tabular}{|l|r|r|r|}
\hline $\begin{array}{l}\text { Rastrigin } \\
\text { dim: 20 }\end{array}$ & PSO & PSO Multi & p-value \\
\hline $\begin{array}{l}\text { Mean CF } \\
\text { Value: }\end{array}$ & $3.283 \mathrm{E}+01$ & $\mathbf{1 . 9 8 3 E}+\mathbf{0 1}$ & $5.478 \mathrm{E}-06$ \\
\hline Std. Dev.: & $8.882 \mathrm{E}+00$ & $6.411 \mathrm{E}+00$ & \\
\cline { 1 - 2 } $\begin{array}{l}\text { CF Value } \\
\text { Median: }\end{array}$ & $3.129 \mathrm{E}+01$ & $1.841 \mathrm{E}+01$ & \\
\cline { 1 - 2 } $\begin{array}{l}\text { Max, CF } \\
\text { Value: }\end{array}$ & $5.981 \mathrm{E}+01$ & $3.482 \mathrm{E}+01$ & \\
\cline { 1 - 2 } $\begin{array}{l}\text { Min, CF } \\
\text { Value: }\end{array}$ & $1.929 \mathrm{E}+01$ & $7.960 \mathrm{E}+00$ \\
\cline { 1 - 2 }
\end{tabular}

Table 12. Results - Rosenbrock function: dim: 20 .

\begin{tabular}{|c|c|c|c|}
\hline $\begin{array}{l}\text { Rosenbrock } \\
\text { dim: } 20\end{array}$ & PSO & PSO Multi & p-value \\
\hline $\begin{array}{l}\text { Mean CF } \\
\text { Value: }\end{array}$ & $1.955 \mathrm{E}+01$ & $1.563 \mathrm{E}+01$ & $2.475 \mathrm{E}-06$ \\
\hline Std. Dev.: & $1.573 \mathrm{E}+00$ & $1.700 \mathrm{E}+00$ & \\
\hline $\begin{array}{l}\text { CF Value } \\
\text { Median: }\end{array}$ & $1.963 \mathrm{E}+01$ & $1.502 \mathrm{E}+01$ & \\
\hline $\begin{array}{l}\text { Max, CF } \\
\text { Value: }\end{array}$ & $2.337 \mathrm{E}+01$ & $1.950 \mathrm{E}+01$ & \\
\hline $\begin{array}{l}\text { Min, CF } \\
\text { Value: }\end{array}$ & $1.583 \mathrm{E}+01$ & $1.351 \mathrm{E}+01$ & \\
\hline
\end{tabular}

\section{The scaling comparison}

In this section, the comparison of scalabilities of the methods is presented. The mean results for three different dimensional settings $(5,10$ and 20) are compared in Figures $1-4$.

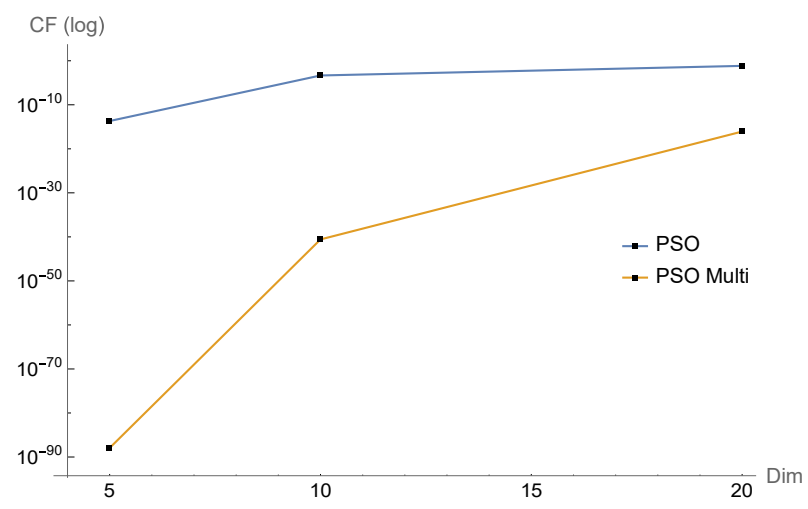

Figure 1. Scaling comparison - Sphere function.

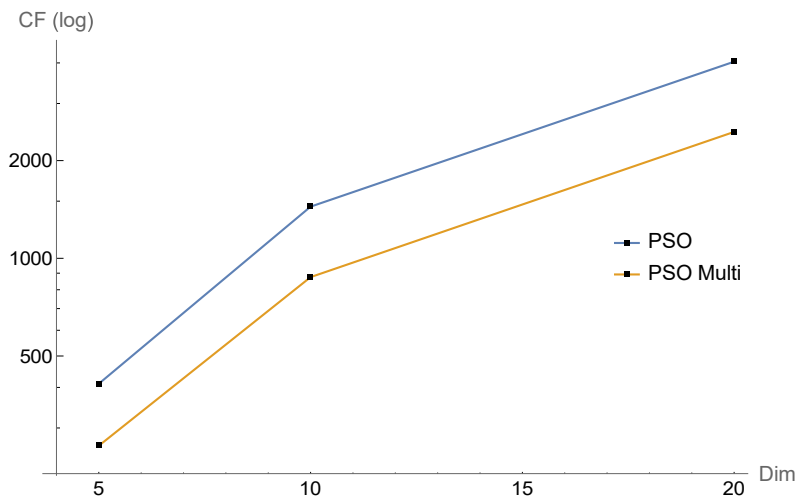

Figure 2. Scaling comparison - Schwefel's function.

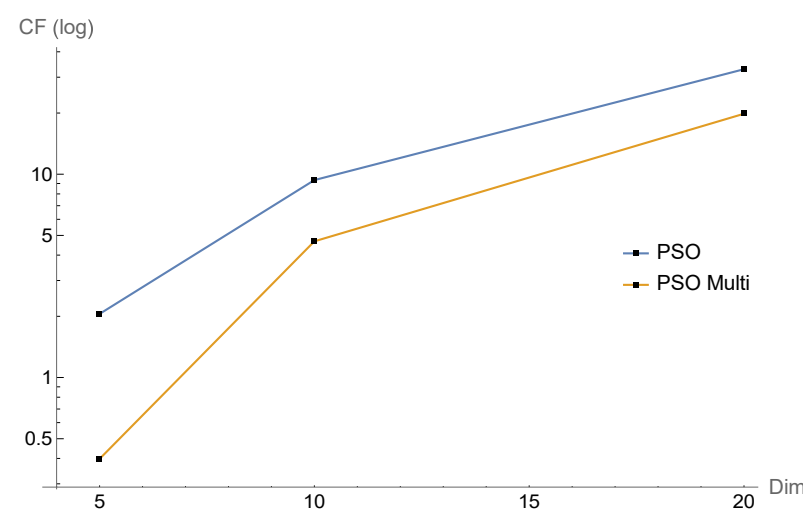

Figure 3. Scaling comparison - Rastrigin's function.

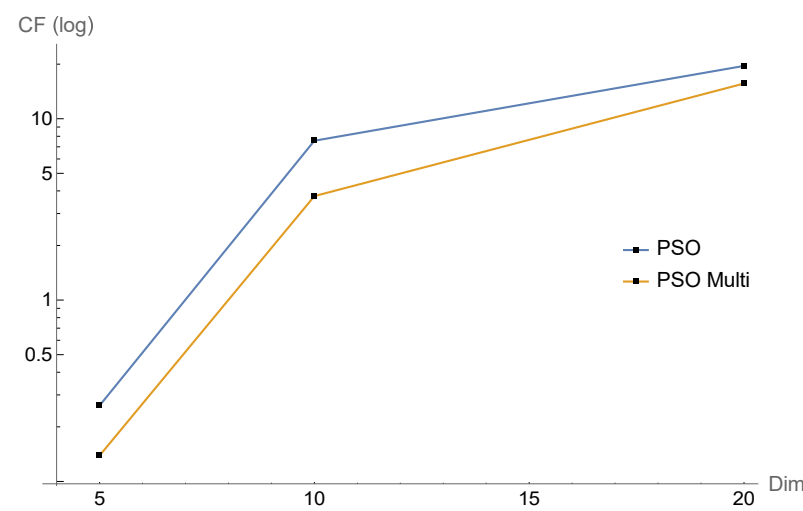

Figure 4. Scaling comparison - Rosenbrock's function.

\section{Results and discussion}

According to data presented in Tables $1-12$ the Multiswarm variant performance is better for all four different test functions in all dimensional settings. The results are statistically significant according to the Wilcoxon signed-rank test. It is worth noting that the results are in many cases significantly better despite that both algorithms utilize the similar total number of particles and the number of iterations.

As is presented in the scalability comparison (Figures $1-4)$ the difference in performance is usually getting smaller with increasing dimensionality of the problem. The performance in higher dimensions will be addressed in future studies.

\section{Conclusions}

In this paper, a comparison of a single swarm and multiswarm PSO was presented. Based on the previous works of (García-Nieto and Alba, 2012) the swarm size for the multi-swarm approach was set to 6 particles. It has been presented that such algorithm is capable of obtaining very good results on four typically used benchmark functions. Also, the performance was in all cases superior to the performance of single swarm PSO algorithm.

The results are surprisingly consistent, and this initial study will be followed by a more detailed study with 
multiple population size settings and more test functions.

Based on the initial results it seems that using basic multi-swarm approach in opposite to a single swarm might be beneficial for PSO based algorithms. The future research will focus on multi-swarm approaches for advanced PSO modifications.

\section{Acknowledgements}

This work was supported by the Ministry of Education, Youth and Sports of the Czech Republic within the National Sustainability Programme Project no. LO1303 (MSMT-7778/2014), further by the European Regional Development Fund under the Project CEBIA-Tech no. CZ.1.05/2.1.00/03.0089 and by Internal Grant Agency of Tomas Bata University under the Projects no. IGA/CebiaTech/2018/003. This work is also based upon support by COST (European Cooperation in Science \& Technology) under Action CA15140, Improving Applicability of Nature-Inspired Optimisation by Joining Theory and Practice (ImAppNIO), and Action IC1406, High-Performance Modelling and Simulation for Big Data Applications (cHiPSet). The work was further supported by resources of A.I.Lab at the Faculty of Applied Informatics, Tomas Bata University in Zlin (ailab.fai.utb.cz).

\section{References}

A. El Dor, M. Clerc, and P. Siarry. A multi-swarm PSO using charged particles in a partitioned search space for continuous optimization. Computational Optimization and Applications, 53(1): 271-295, 2012.

A. Engelbrecht. Heterogeneous particle swarm optimization. In Proceedings of the 7th international conference on Swarm intelligence. Berlin, Heidelberg: Springer-Verlag, pages 191-202, 2010.

J. García-Nieto, E. Alba. Why six informants is optimal in PSO. In Proceedings of the 14th annual conference on Genetic and evolutionary computation, pages 25-32, ACM. 2012.

J. Kennedy and R. Eberhart. Particle swarm optimization. In Proceedings of the IEEE International Conference on Neural Networks, pages 1942-1948, 1995.

J. Kennedy. The particle swarm: social adaptation of knowledge. In Proceedings of the IEEE International Conference on Evolutionary Computation, pages 303-308, 1997.

J.-J. Liang and P.N. Suganthan. Dynamic multi-swarm particle swarm optimizer with local search. In Evolutionary Computation, The 2005 IEEE Congress on. IEEE, 2005. pages 522-528, 2005.

Y. Liu, G. Wang, H. Chen, H. Dong, X. Zhu, and S. Wang. An improved particle swarm optimization for feature selection. Journal of Bionic Engineering, 8(2): 191-200, 2011.

L. Ostadrahimi, A. M.M iguel, and A. Abbas. Multi-reservoir operation rules: multi-swarm PSO-based optimization approach. Water resources management, 26(2): 407-427, 2012.

S. Solomon, P. Thulasiraman, and R. Thulasiram. Collaborative multi-swarm PSO for task matching using graphics processing units. In Proceedings of the 13th annual conference on Genetic and evolutionary computation, pages 1563-1570, ACM. 2011.

Y. Shi and R. Eberhart. A modified particle swarm optimizer. In Proceedings of the IEEE International Conference on Evolutionary Computation (IEEE World Congress on Computational Intelligence), pages 69-73. I. S. 1998.

F. van den Bergh and A.P. Engelbrecht. A study of particle swarm optimization particle trajectories. Information Sciences, 176(8): 937-971, 2006. 